\title{
Numerical and experimental demonstration of a wavelength demultiplexer design by point-defect cavity coupled to a tapered photonic crystal waveguide
}

\author{
Zeki Hayran, ${ }^{1}$ Mirbek Turduev, ${ }^{2, *}$ Muriel Botey, ${ }^{3}$ Ramon Herrero, ${ }^{3}$ \\ Kestutis Staliunas, ${ }^{3,4}$ and Hamza Kurt ${ }^{1}$ \\ ${ }^{1}$ Nanophotonics Research Laboratory, Department of Electrical and Electronics Engineering, \\ TOBB University of Economics and Technology, Ankara 06560, Turkey \\ ${ }^{2}$ Department of Electrical and Electronics Engineering, TED University, Ankara 06420, Turkey \\ ${ }^{3}$ DONLL, Departament de Física, Universitat Politècnica de Catalunya (UPC), Edifici Gaia, Rambla Sant Nebridi 22, 08222 Terrassa, Spain \\ ${ }^{4}$ Institució Catalana de Recerca i Estudis Avancats (ICREA), Passeig Lluís Companys 23, 08010 Barcelona, Spain \\ *Corresponding author: mirbek.turduev@tedu.edu.tr
}

Received 25 September 2015; revised 12 November 2015; accepted 14 November 2015; posted 16 November 2015 (Doc. ID 250748); published 21 December 2015

We propose and experimentally demonstrate a demultiplexer with point-defect resonators and a reflection feedback mechanism in a photonic crystal waveguide (PCW). A tapered PCW has been chosen as the necessary reflector, which enhances the drop efficiency. Due to the variation of the single-mode waveguide width of the tapered PCW, spatial alteration of the effective refractive index can be achieved. This phenomenon is used to reflect back the forward propagating wave which is then coupled again to the drop channels via the resonators. High transmission efficiency to the dropout channels is numerically predicted by calculations, either in two- and three-dimensional models, and analytically described by a coupled-mode theory. Moreover, an experimental realization in the microwave regime provides confirmation that the targeted wavelengths can be properly transmitted at the drop channels with low crosstalk and relatively high efficiencies. () 2015 Optical Society of America

OCIS codes: (130.0250) Optoelectronics; (160.5298) Photonic crystals; (230.7380) Waveguides, channeled; (230.7408) Wavelength filtering devices; (250.5300) Photonic integrated circuits; (350.4010) Microwaves.

http://dx.doi.org/10.1364/OL.41.000119

Optical signal processing requires monitoring wavelengths of light that propagate in an optical waveguide. While the main channel operates as a broadband waveguide, an efficient demultiplexing is often required, such that the targeted wavelengths are directed toward the drop channels with low crosstalk and efficient coupling. The spacing between drop channels also plays an important role in monitoring a large number of wavelengths, while small size is essential for these devices.
Such compact wavelength de-multiplexing (WDM) devices can be built with the so-called Bragg gratings [1,2], which show a wavelength dependent reflection. However, each proposal has inherent difficulties, resulting in the limited experimental realization in a small-scale compact device. The difficulty of realization of such devices at a very small scale gathered the attention to WDM devices based on photonic crystals (PCs), which could be suited for ultra-dense integration.

Some of the demultiplexing studies are based on the superprism effect observed in PCs, where the dispersive phenomena enable high angle-sensitive light propagation, but with limited wavelength resolution. Moreover, spatially broadening of the propagating beam inside the structure requires large dimensions that render the devices to be less compact. Furthermore, in the superprism-based demultiplexing devices, the incident beam's spatial profile becomes distorted, eventually limiting the coupling efficiency to the output channels [3-6]. Another powerful approach for WDM purposes is to spatially separate the wavelengths of the input light along a PCW. For instance, a four-channel coarse WDM device based on the modification of the air holes occupying the sides of the waveguide has been proposed in Ref. [7]. Another study demonstrated wavelength monitoring, by utilizing the mini stop band phenomena [8]. On the other hand, PC channel drop filters, based on point or line defect cavities, have also attracted attention due to their high quality factor, high drop efficiency, and feasible fabrication at the micron scale [9]. So far, ideal transfer characteristics utilizing parallel waveguides and a resonator in between have been demonstrated by forcing the resonator to support two degenerate modes [10-12]. On the other hand, point defects coupled to a waveguide lateral to the bus waveguide have also been investigated. Theoretical $100 \%$ drop efficiency has been derived 
in the presence of a reflection feedback formed by a closed waveguide [13] and wavelength selective resonator [14].

In this Letter, we propose a tapered PC waveguide, accompanied by side-coupled cavities that are tuned to specific wavelengths. While the cavities are responsible for selecting the desired wavelengths, the tapered waveguide acts as reflection feedback with cutoff frequencies depending on the penetration depth. The dispersion of the waveguide smoothly changes, along with its width. As the width decreases along the propagation direction, its cutoff frequency increases. Therefore, higher frequencies are allowed to propagate farther in the waveguide compared to lower frequencies which are reflected back. The wavelength selectivity was numerically verified with the finite-difference time domain (FDTD) method in two and three dimensions (2D/3D) [15]. Furthermore, an experimental demonstration of the idea in the microwave region was performed, showing good agreement with the 3D FDTD results.

It is well known that a PC waveguide can confine and guide an incoming wave at a specific frequency range. The "bottom frequency" (the cutoff frequency) depends on the design parameters of the PC waveguide. Therefore, by systematically engineering the design parameters along the propagation direction, one can obtain a gradual cutoff frequency variation. Here, we change the waveguide in a linear way by tilting the upper and lower sides of the PC waveguide structure clockwise and anticlockwise, respectively. Throughout this Letter, a rotation angle of $\theta=0.40^{\circ}$ has been used in all numerical and experimental analyses. The tilt angle was chosen so that the cutoff frequencies of the waveguide comprise the operational frequency span within its length. Figure 1(a) shows the schematic representation of the proposed design. The PCs are made up of a square lattice of dielectric alumina $\left(\mathrm{Al}_{2} \mathrm{O}_{3}\right)$ rods, whose dielectric constant is equal to $\varepsilon_{r}=9.61$. The lattice constant " $a$ " is set to $10 \mathrm{~mm}$, and the radius of the dielectric rods is equal to $r=0.317 a=$ $3.17 \mathrm{~mm}$. The rotation angle of $\theta=0.40^{\circ}$ results in a linearly varied width of the channel, from $W_{1}=1.98 a=19.8 \mathrm{~mm}$ down to $W_{2}=1.42 a=14.2 \mathrm{~mm}$, while the structural length is set equal to $L_{x}=38 a=380.0 \mathrm{~mm}$. The extraction of the desired frequency components is achieved by creating a point defect coupled to a line defect whose orientation is perpendicular to the main channel. As a demonstration, three channels have been designed with a channel spacing of $4 a$ and $5 a$. It has been found that this kind of spacing is sufficient to avoid the effects of coupling between the point defect resonators. The point defect resonators are created by removing a single rod, and their respective drop waveguides are formed by removal of single row of rods. The single rod is removed at the third innermost row of the PC waveguide so that two rods are located between the point defect and the main waveguide. The resonators have been tuned to the desired resonance frequencies by changing the size of the point defect, moving the innermost rods either inward or outward. As depicted in Figs. 1(b) and 1(c), the four innermost dielectric rods of the first and third channels were displaced at a distance equal to $0.03 a=0.30 \mathrm{~mm}$, outward and inward, with respect to the second cavity, either to decrease or increase the resonance frequencies, while the rod of the second cavity remains unchanged. It is worth noting that the distance between the input channel and the three drop channels is 3,8 , and $12 a(30,80$, and $120 \mathrm{~mm}$ ), respectively.

Using coupled-mode theory (CMT) in time domain, an analytical approach can be used to investigate the transmission

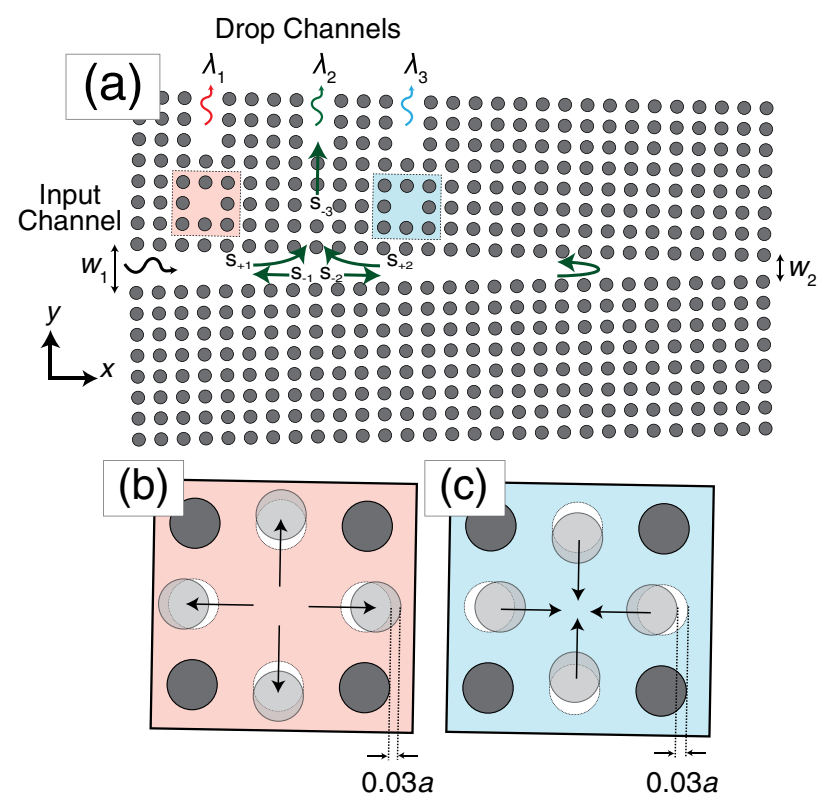

Fig. 1. (a) Schematic representation of the proposed structure. Wave amplitudes are exemplified for the second channel; $S_{+1}$ and $S_{+2}$ are the amplitudes of the incoming waves, whereas $S_{-1}, S_{-2}$, and $S_{-3}$ are the amplitudes of the outgoing waves in each port. The cavity regions of the first and third channels are shown in light red and light blue, respectively. The locations of the innermost rods of these regions have been altered to change the resonance frequency of the (b) first and (c) third channels, whereas the cavity region of the second channel remained unchanged. The arrows in (b) and (c) indicate the directions (outward or inward) in which the rods were moved.

spectra at the drop channels. By employing time-reversal symmetry and power conservation and taking the Fourier transform of the respective wave amplitude equations $[13,14,16]$, the transmission efficiency for a single drop channel as a function of angular frequency $\omega$ can be derived as

$$
\begin{aligned}
|T(\omega)| & =\frac{\left|S_{-3}(\omega)\right|^{2}}{\left|S_{+1}(\omega)\right|^{2}} \\
& =\left|\frac{\sqrt{\frac{4}{\tau_{3}}}\left(\sqrt{\frac{1}{\tau_{1}}}+\sqrt{\frac{1}{\tau_{2}}} \exp (-i \phi(\omega))\right)}{i\left(\omega-\omega_{0}\right)+\frac{1}{\tau_{1}}+\frac{1}{\tau_{3}}+\frac{1}{\tau_{2}}(1+2 \exp (-i \phi(\omega)))}\right|^{2},
\end{aligned}
$$

where $S_{-3}(\omega)$ and $S_{+1}(\omega)$ are, respectively, the outgoing wave amplitude in the frequency domain at the drop channel and the incoming wave amplitude at the input main channel, which are also shown as an inset in Fig. 1(a). The terms in Eq. (1) $1 / \tau_{1}$, $1 / \tau_{2}$, and $1 / \tau_{3}$ denote the decay rates in the input side of the main channel, the reflection side of the main channel, and the drop channel, respectively, while $\omega_{0}$ is the resonance frequency of the PC cavity, and $\varphi(\omega)$ is the phase retardation due to the frequency dependent reflection at the main channel. Equation (1) provides insight about the physical mechanism and operating principle of the idea presented in this Letter. One of the foremost conclusions based on the CMT model is that the transmission efficiency can be maximized by a proper selection of the decay rates, $1 / \tau_{1,2,3}$, and phase retardation, $\varphi(\omega)$; this has already been studied in detail for different feedback mechanisms 


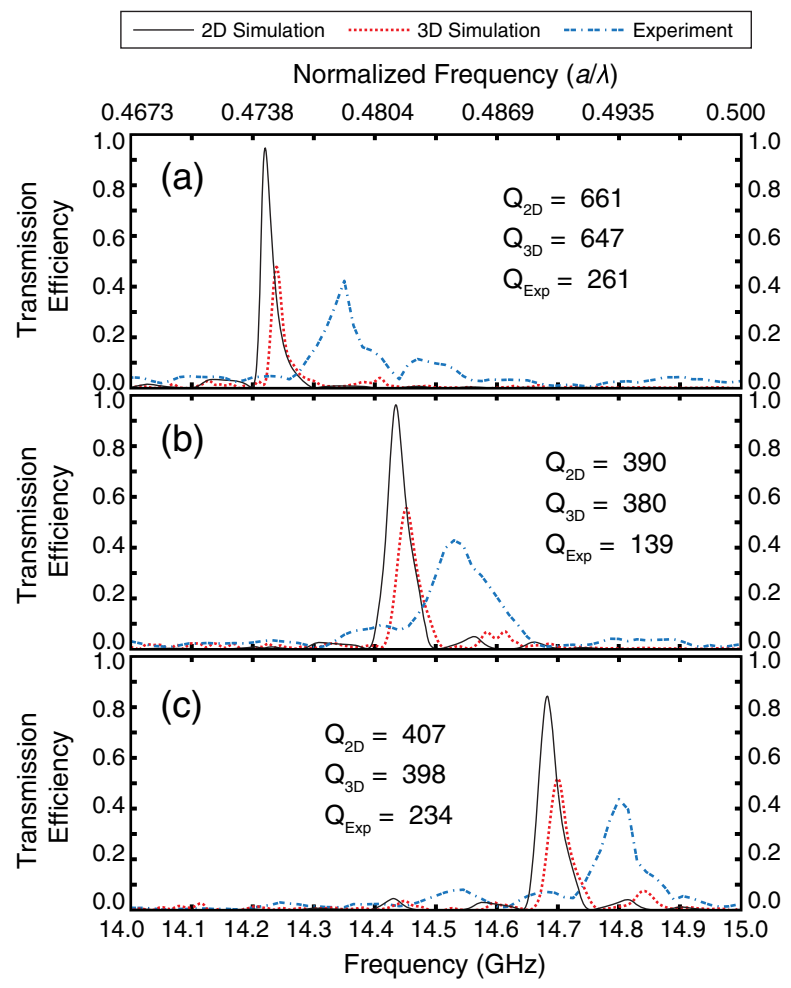

Fig. 2. Calculated and measured transmission spectra for the (a) first, (b) second, and (c) third channels, with a channel spacing equal to $5 a$ and $4 a .2 \mathrm{D}$ and 3D FDTD calculations along with the experimental result are presented together. Normalized frequencies are converted into actual values in the $\mathrm{GHz}$ range. For each spectrum, the corresponding quality factors $\mathrm{Q}_{2 \mathrm{D}}, \mathrm{Q}_{3 \mathrm{D}}$, and $\mathrm{Q}_{\operatorname{Exp}}$ are given for the $2 \mathrm{D}$ simulation, $3 \mathrm{D}$ simulation, and experiment, respectively.

$[13,14]$. In this regard, to optimize the coupling between the waveguide and the resonators, the resonators are precisely placed at the positions where the phase delay is nearly an integer multiply of $2 \pi$ for the respective resonant wavelengths.

To verify the operational principle, FDTD methods in 2D and $3 \mathrm{D}$ were performed for transverse magnetic (TM) polarized light (electric field has only out-of-plane components, $\mathrm{E}_{z}$ ), where numerical calculations in $3 \mathrm{D}$ also allowed us to observe the issue of vertical light confinement behavior. Perfectly matched boundary conditions were used as an absorbing boundary to terminate the computational domain. A plane wave with a spatial width equal to $1 a=10 \mathrm{~mm}$ and a longitudinal distance equal to $0.5 a=5 \mathrm{~mm}$ with respect to the input was launched into the main waveguide. Detectors were placed in the drop waveguides to determine the corresponding spectral field profile of the drop channels.

The calculated transmission spectra for the first, second, and third channels are shown in Figs. 2(a)-2(c), respectively. Drop efficiencies larger than $95 \%$ for the first and second channels, and larger than $80 \%$ for the third channel, were achieved with the $2 \mathrm{D}$ model. The corresponding crosstalks for each channel are $-22.0,-13.6$, and $-17.9 \mathrm{~dB}$, respectively. For both cases, the structural height is set to $L_{z}=15.5 a=155.0 \mathrm{~mm}$. Figure 2 indicates that the out-of-plane losses occur for all channels in $3 \mathrm{D}$ simulations. In this case, drop efficiencies vary between $45 \%$ and $55 \%$. Moreover, even though the 3D simulated efficiency is smaller than the $2 \mathrm{D}$, still similar crosstalks such as $-16.5,-11.8$, and $-16.8 \mathrm{~dB}$ were predicted for the first, second, and third channels, respectively. A slight shift of the transmission curves also occurs in the frequency axis. This is expected as the $2 \mathrm{D}$ analysis ideally assumes infinitely long dielectric rods. Limited heights of the rods considered in 3D provide an effective index of the dielectric material that can be slightly different from that of the 2D case. Moreover, as can be deduced from Eq. (1), the angular frequency $\omega$ that maximizes the transmittance through the channels depends not only on the resonance frequency, $\omega_{0}$, but also on the decay rate, $1 / \tau_{2}$, and phase retardation, $\varphi(\omega)$. In this regard, one can conclude that the shifting of the resonance frequency may be also attributed to deviations in those terms.

For an experimental demonstration at the microwave region, we used dielectric alumina rods, as in numerical calculations. Figures 3(a) and 3(b) show the schematics and photographic illustration of the experimental setup, respectively. A vector network analyzer, Agilent E5071C ENA, was used to obtain the transmission efficiency data. Horn antenna pairs that are shown in Fig. 3(b) as receiver and transmitter antennas, with an operating frequency range between $12-15 \mathrm{GHz}$, were employed for transmitting and receiving the input and output signals, respectively. We carefully matched the numerical and experimental excitation conditions by placing the source with a distance equal to $0.5 a=5 \mathrm{~mm}$ from the entrance of the waveguide in both cases. Moreover, microwave absorbers are placed around the structure to reduce possible back reflections, as can be seen in Fig. 3(b). A TM-polarized wave was launched into the waveguide; the detected power was normalized by dividing it into the detected power in a waveguide of a constant width, which is equal to the width of the tapered waveguide at the entrance. The obtained experimental transmission efficiency for each channel is superimposed in Figs. 2(a)-2(c). A good match between the 3D simulated model and experimental realization is observed. The measured crosstalks for each channel are $-12.2,-7.3$, and $-8.5 \mathrm{~dB}$. These values deviate from the $3 \mathrm{D}$ numerically calculated crosstalks which are $-16.5 \mathrm{~dB},-11.8 \mathrm{~dB}$, and $-16.8 \mathrm{~dB}$, respectively. The quality factors of the resonant modes are lower than the numerical simulations. The difference is mainly due to material absorption [17] and fabrication imperfections [18].

Further investigations were made by replacing the receiver horn antenna with a monopole antenna to record the steadystate intensity distribution along the main waveguide. The intensity distribution was obtained with the monopole antenna by measuring the evanescent field intensity at the top of the constructed structure. The monopole antenna was placed perpendicular to the surface rods $(x-y$ plane), and the tip of the antenna was inserted $10 \mathrm{~mm}$ into the main waveguide. By moving the monopole antenna with spatial steps equal to $2 \mathrm{~mm}$ along the middle of the main waveguide, the crosssectional intensity distribution along the main waveguide was measured. Figure 4(a) first shows the steady-state intensity distribution obtained from 2D numerical calculations (see the animation of the light coupling behavior to the dropout channels for three different operating wavelengths in Visualization 1). It is seen that the first drop channel effectively selects the input radiation via resonant effect of the cavity. The intensity profile along the dotted line [inset in Fig. 4(a)] is given in Fig. 4(b), along with the experimental result of the intensity distribution. The numerical result is obtained from 
Photonic Crystal De-Multiplexer

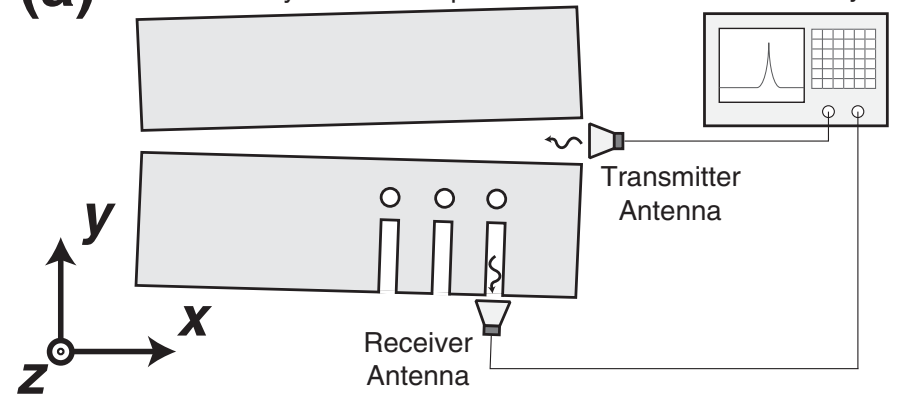

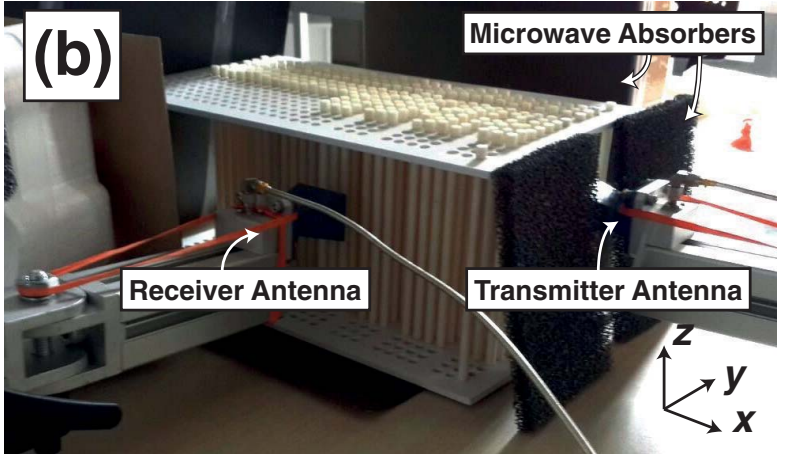

Fig. 3. (a) Schematic and (b) photographic representation of the experimental setup used to investigate the proposed photonic crystal wavelength demultiplexer at the microwave regime.

the 3D FDTD calculations by extracting the intensity profile at the same height where the monopole antenna has taken the measurements. On the other hand, the numerical result obtained from the 2D FDTD calculations is also sub-plotted in Fig. 4(b), and the longitudinal position of the resonator is marked with a red dashed line to show the phase delay of the incoming wave at the respective resonator position. The difference of the phase delays between $2 \mathrm{D}$ and $3 \mathrm{D}$ analyses is calculated as $0.18,0.06$, and $0.19 \pi$ for the first, second, and third channels, respectively; this can be considered as a weak perturbation. Therefore, one can deduce that the transmission efficiency losses in the 3D cases are dominated by the propagation losses, rather than the phase condition mismatches. Hence, out-of-plane losses do not seem to play an important role due to a relatively large height of the structure and short propagation distance. Moreover, as the propagation distance becomes larger, the spatial oscillation of the intensity distribution increases. This observation is valid for both numerical and experimental
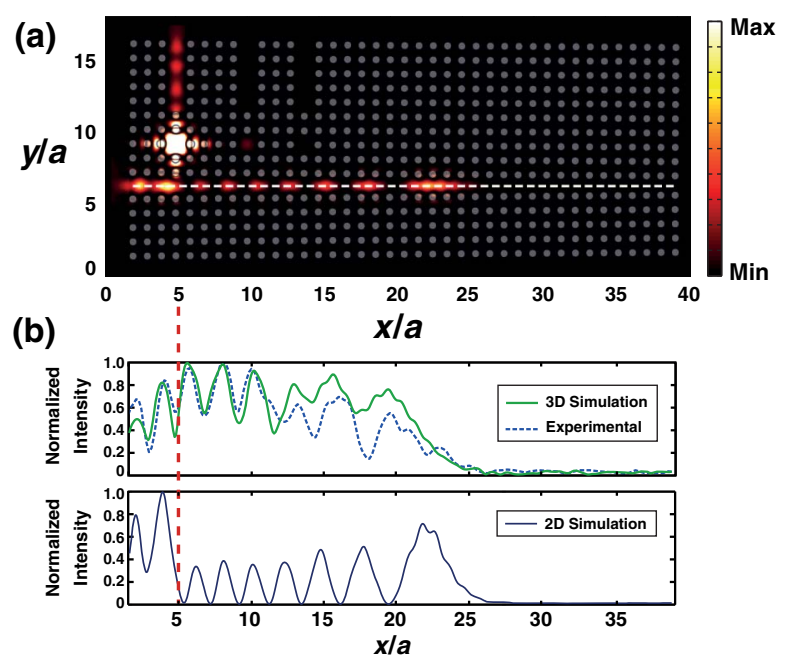

Fig. 4. (a) Steady-state intensity distribution of the proposed demultiplexer at the resonance frequency of the first channel, which corresponds to $14.22,14.24$, and $14.35 \mathrm{GHz}$ for 2D FDTD, 3D FDTD, and the experimental case, respectively. In addition, light coupling animation for the all three operating wavelengths has been prepared (see Visualization 1). (b) 3D numerical and experimental cross-sectional intensity profile along the propagation direction at the bus waveguide, sub-plotted with the $2 \mathrm{D}$ numerical results. results as is expected from the decreasing group velocity caused by the gradual waveguide width alteration. Toward the end of the tapered waveguide, the mode approaches cutoff frequency and, thus, becomes highly dispersive.

In summary, we have proposed an all-dielectric PC-based wavelength demultiplexer and verified its operational principle both numerically and experimentally. A crosstalk smaller than $12 \mathrm{~dB}$ has been experimentally achieved in a three-channel configuration. We should note that the proposed all-dielectric demultiplexer structure is relatively compact and experimentally feasible for fabrication. Furthermore, crosstalks can also be reduced either by implementing higher $Q$ cavities or increasing the distances between the drop channels.

Acknowledgment. H. Kurt acknowledges partial support of the Turkish Academy of Science.

\section{REFERENCES}

1. G. P. Agrawal and S. Radic, IEEE Photon. Technol. Lett. 6, 995 (1994).

2. R. Zengerle and O. Leminger, J. Lightwave Technol. 13, 2354 (1995).

3. H. Kosaka, T. Kawashima, A. Tomita, M. Notomi, T. Tamamura, T. Sato, and S. Kawakami, Appl. Phys. Lett. 74, 1370 (1999).

4. K. B. Chung and S. W. Hong, Appl. Phys. Lett. 81, 1549 (2002).

5. B. Momeni, J. Huang, M. Soltani, M. Askari, S. Mohammadi, M. Rakhshandehroo, and A. Adibi, Opt. Express 14, 2413 (2006).

6. T. Matsumoto, T. Asatsuma, and T. Baba, Appl. Phys. Lett. 91, 091117 (2007).

7. T. Niemi, L. H. Frandsen, K. K. Hede, A. Harpoth, P. I. Borel, and M. Kristensen, IEEE Photon. Technol. Lett. 18, 226 (2006).

8. E. Viasnoff-Schwoob, C. Weisbuch, H. Benisty, C. Cuisin, E. Derouin, O. Drisse, G. H. Duan, L. Legouezigou, O. Zegouezigou, F. Pommereau, S. Golka, H. Heidrich, H. J. Hensel, and K. Janiak, Appl. Phys. Lett. 86, 101107 (2005).

9. S. Noda, A. Chutina, and M. Imada, Nature 407, 608 (2000).

10. S. Fan, P. R. Villeneuve, J. D. Joannopoulos, and H. A. Haus, Phys. Rev. Lett. 80, 960 (1998).

11. S. Fan, P. R. Villeneuve, J. D. Joannopoulos, and H. A. Haus, Opt. Express 3, 4 (1998).

12. B. K. Min, J.-E. Kim, and H. Yong Park, Opt. Commun. 237, 59 (2004).

13. S. Kim, I. Park, H. Lim, and C. Kee, Opt. Express 12, 5518 (2004).

14. H. Ren, C. Jiang, W. Hu, M. Gao, and J. Wang, Opt. Express 14, 2446 (2006).

15. Lumerical Solutions "FDTD Solutions," https://www.lumerical.com/.

16. H. A. Haus, Waves and Fields in Optoelectronics (Prentice-Hall, 1984).

17. T. Xu, M. S. Wheeler, H. E. Ruda, M. Mojahedi, and J. S. Aitchison, Opt. Express 17, 8343 (2009).

18. T. Asano, B. S. Song, and S. Noda, Opt. Express 14, 1996 (2006). 